Document downloaded from:

http://hdl.handle.net/10251/63715

This paper must be cited as:

Gómez-Tejedor, JA.; Castro Palacio, JC.; Monsoriu Serra, JA. (2014). Direct measurement of the speed of sound using a microphone and a speaker. Physics Education. 49(3):310313. doi:10.1088/0031-9120/49/3/310.

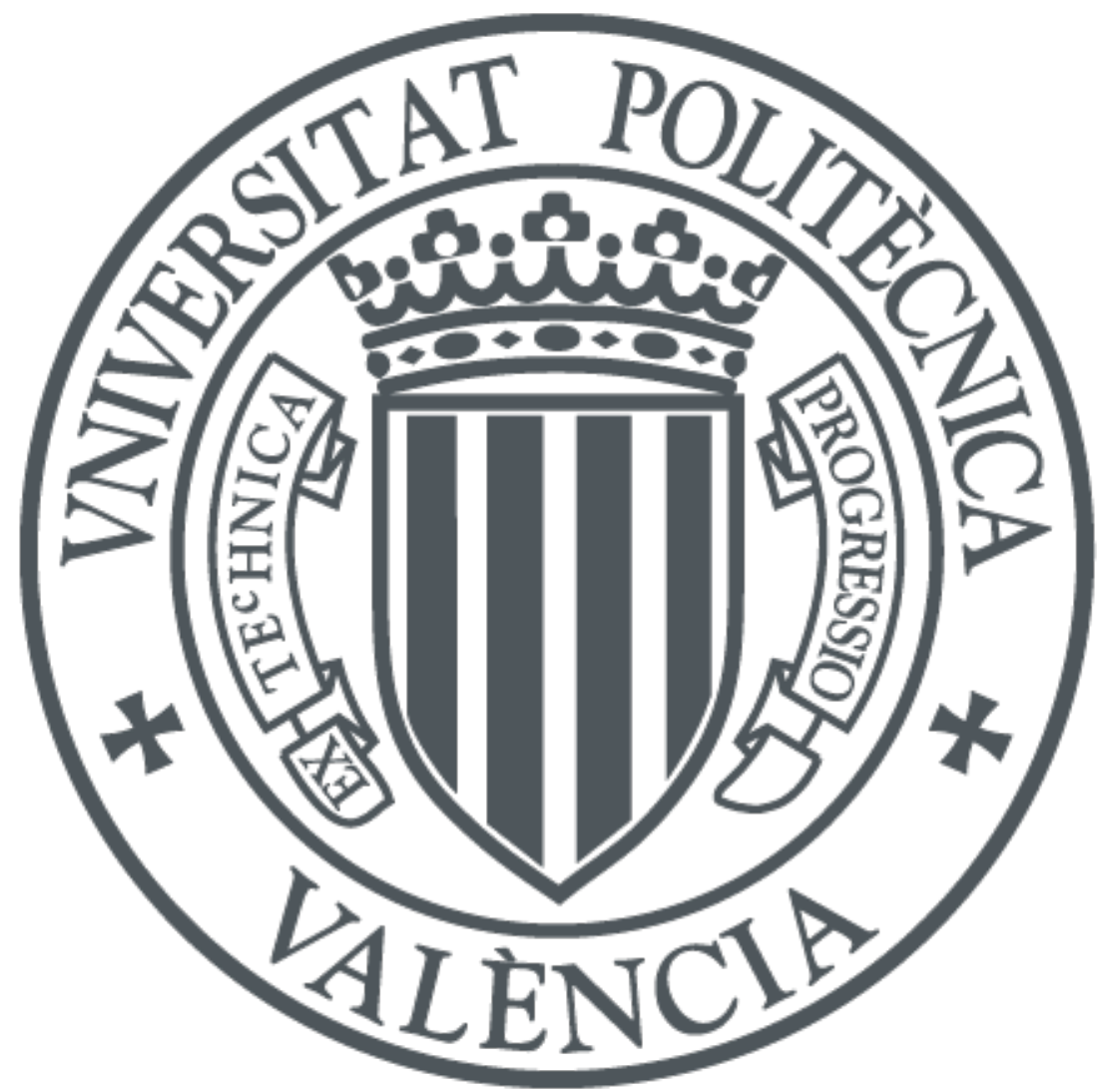

The final publication is available at

http://dx.doi.org/10.1088/0031-9120/49/3/310

Copyright IOP Publishing: Hybrid Open Access

Additional Information 


\title{
Direct measurement of the speed of sound by using a microphone and a speaker
}

\author{
José Antonio Gómez-Tejedor \\ Centro de Biomateriales e Ingeniería Tisular, Universitat Politècnica de València, \\ Camí de Vera s/n, 46022, València, Spain. \\ E-mail: jogomez@fis.upv.es
}

\section{Juan Carlos Castro-Palacio}

Departement Chemie, Physikalische Chemie, Universität Basel, Klingelbergstrasse 80, CH-4056 Basel, Switzerland.

E-mail: juancarlos.castropalacio@unibas.ch

\section{Juan A. Monsoriu}

Centro de Tecnologías Físicas, Universitat Politècnica de València, Camí de Vera s/n, 46022, València, Spain.

E-mail: jmonsori@fis.upv.es

\section{Physics Education 49 (3) 2014: 310-13}

\begin{abstract}
We present a simple and accurate experiment to obtain the speed of sound in air by using a conventional speaker and a microphone connected to a computer. A free open source digital audio editor and recording computer software application allows determining the time-of-flight of the wave for different distances from which the speed of sound is calculated. The result is in very good agreement with the reported value in the literature.
\end{abstract}




\section{Introduction}

Recently, several methods based on standing waves have been proposed to measure the speed of sound in the air[1], water[2] and metal[3] with good accuracy. However, one of the most standard method to measure the speed of sound is the time-of-flight method

[4]. It consists of measuring the speed of sound by dividing the direct measurement of the distance traveled by the wave over the direct measurement of the time period it takes to go from the source to the receptor. For instance, in 1 meter, a sound wave travelling in the air at $346.6 \mathrm{~m} / \mathrm{s}$ takes $0.003 \mathrm{~s}$ [5]. It means that if we want to measure the speed of sound in 1 meter of distance, a resolution for time of less than $0.003 \mathrm{~s}$ should be considered.

Taking into account that electricity is much faster than sound in the air, a suitable way to fulfill the above criterion is by using a speaker and a microphone. In our experimental setup, the speaker is connected to a frequency generator which emits a single sound frequency. Both devices are connected to a computer. The time-of-flight of the wave is measured by means of a computer free software for different relative distances between the speaker and the microphone.

This experiment is very simple to perform by students, and it requires very simple equipment, that can be easily found in every Physics teaching laboratory. We have used a signal generator to generate a sinusoidal sound wave, which can be also generated by the speaker connected to the computer. We have used a professional microphone in order to get accurate data, but the experiment can also be performed with a very cheap microphone. Then, this method provides a very accurate measurement of the speed of sound which can be easily reproduced by the students at High School classrooms or laboratories, by following the procedure described in this paper.

\section{Experiment}

The experimental setup to measure the speed of sound is presented in Figure 1, in panel a the photograph and in panel b, the schematic representation. The speaker ( 1 in Figure) is placed at a certain distance from the microphone ( 2 in Figure). The speaker is connected to a frequency generator, model Agilent 33220A used to generate a sinusoidal signal of frequency $2000 \mathrm{~Hz}$ and amplitude $350 \mathrm{mV}$. This frequency has been chosen since, after several trials, it was the one for which the speaker-microphone system had the best behavior, that is, with less distortion of the signal received by the microphone. The frequency generator is connected to the right channel of the microphone input of the computer. The microphone is connected to the left input channel by means of an audio amplifier based on a chip UA741CN, with a gain factor of 22. By controlling the volume of the speaker, the intensity of the sound signal is increased as the distance to the microphone increases. The speaker is one of those commonly used in commercial computers. The microphone is a professional model DM1003, although a cheaper microphone can also be used. If there is no frequency generator 
(a)

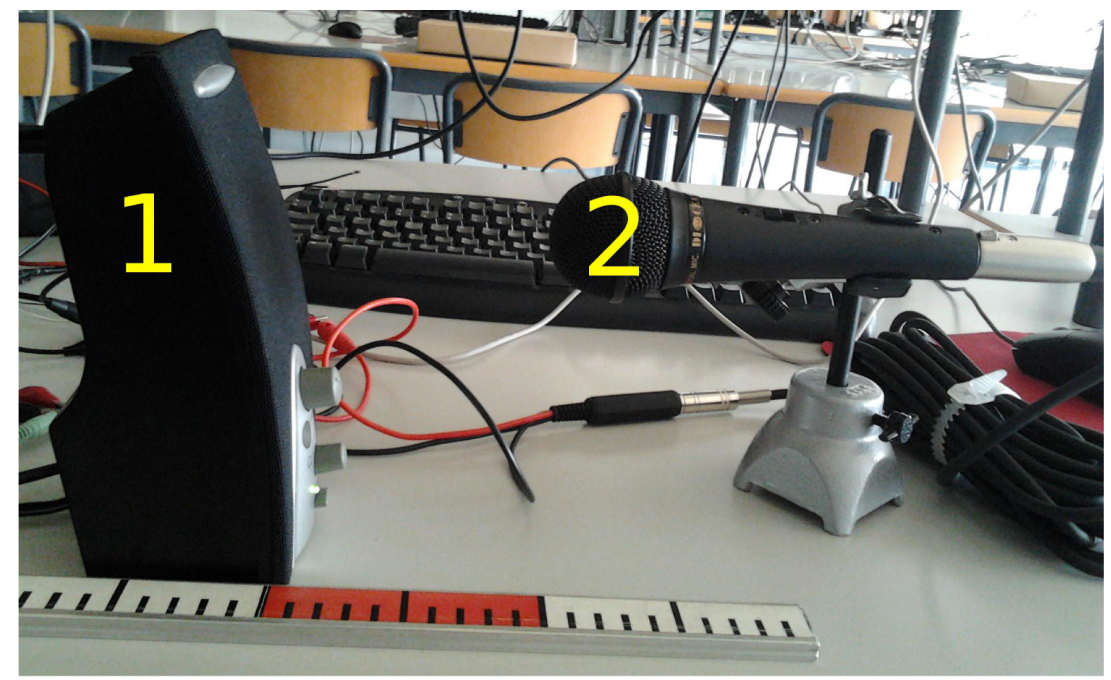

(b)
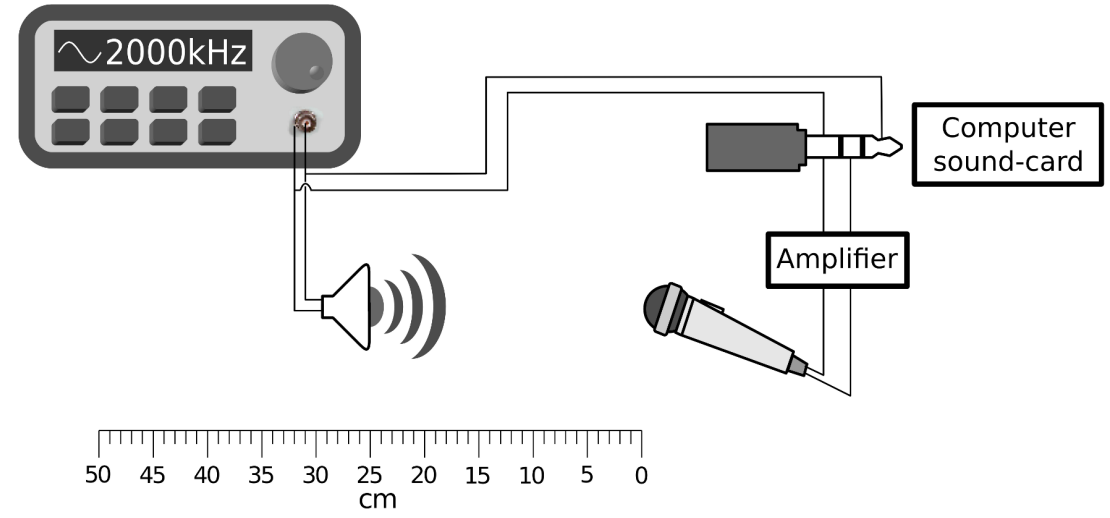

Figure 1. Photograph and schematic representation of the experimental set up used to measure the speed of sound.

available, the speaker can be connected directed to the computer and used to generate a signal of single frequency.

The incoming signal to the microphone is recorded on the left-side channel by using the free application Audacity version 2.0.3 [6] (sound wave at the bottom of Figure 2a and $2 \mathrm{~b}$ ). At the same time, the signal of the frequency generator is recorded by the right-side channel (sinusoidal sound wave at the top of Figure 2a and $2 \mathrm{~b}$ ). Both signals are recorded simultaneously over some seconds in stereo and with a sampling frequency of $44100 \mathrm{~Hz}$. By using the application Audicity, the time difference between the signal emitted by the speaker and the signal received by the microphone is measured. The time difference is measured from the moment at which the signal of the speaker crosses the y-axis at zero (going from positive values to negative values: see points labelled as 1 in Figure 2a and 3 in Figure 2b) until the signal of the microphone does the same (points labelled as 3 in Figure $2 \mathrm{a}$ and 5 in Figure 2b). In Figure 2a the distance between the microphone and the speaker is $20 \mathrm{~cm}$. The result in Figure 2a from point 1 to point 2 takes into account 22 samples (indicated by the Spanish word "muestras" in 

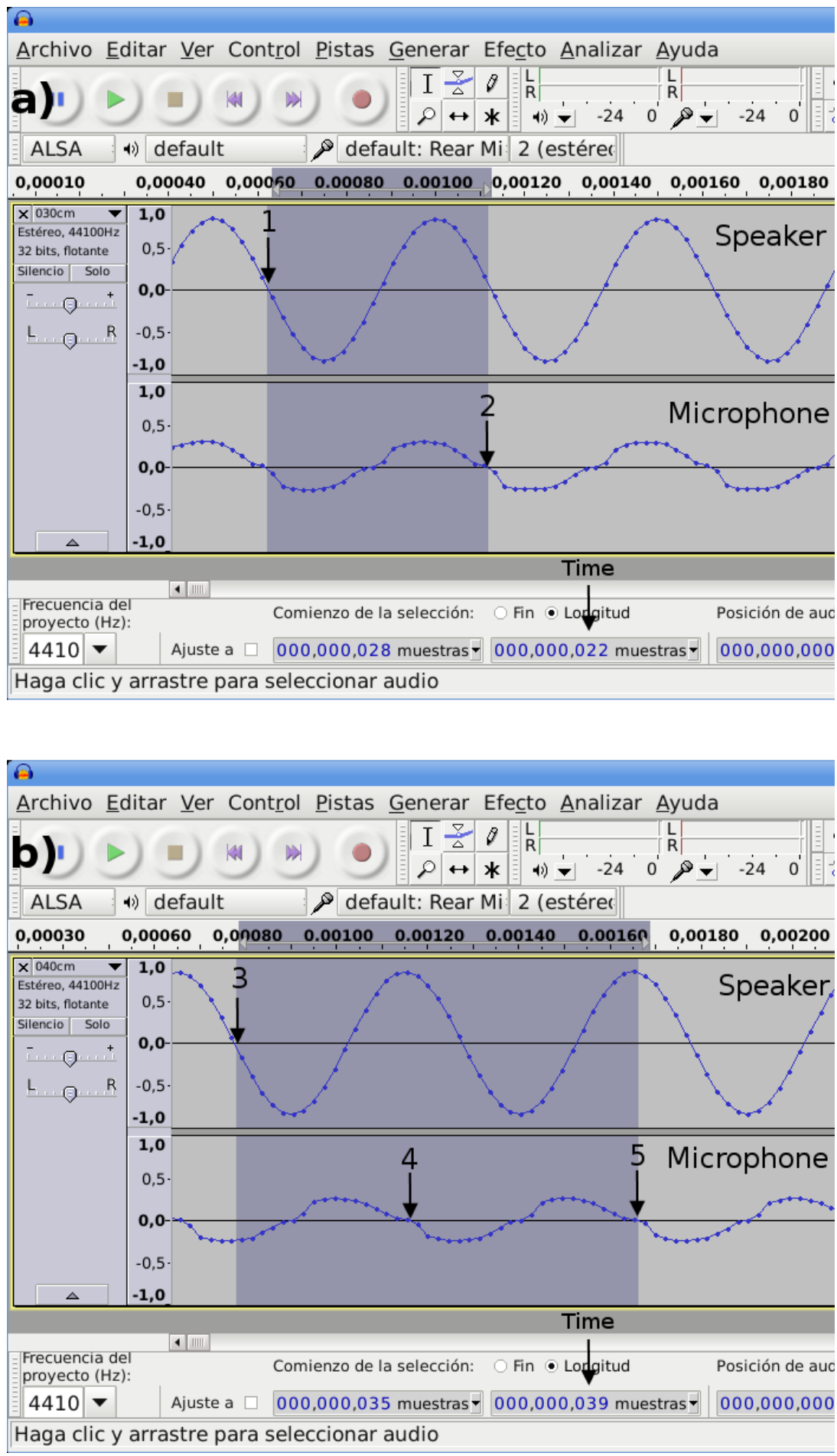

Figure 2. Snapshot of Audacity application with the measurement of the time difference between the signal of the speaker (upper sine wave) and the signal of the microphone (lower sound wave). For these measurements, the distance between the speaker and the microphone were $20 \mathrm{~cm}$ for panel a) and $30 \mathrm{~cm}$ for panel b).

Audacity software, because we are using Spanish version), and each sample corresponds to $(1 / 44100) \mathrm{s}$ which makes a time difference between the signals of $0.499 \mathrm{~ms}$. For the 
case of Figure 2b we have increased the distance to $30 \mathrm{~cm}$. In this case, we can not consider the first time the microphone signal crosses the y-axis (point labelled by 4 in Figure $2 \mathrm{~b}$ ) because this would produce a result of 17 samples, i.e., $0.385 \mathrm{~ms}$ that is a smaller time than the previous one, which is not possible because we have increased the distance. Then, in this case it is necessary to consider the second time that the signal crosses the y-axis (point labelled by 5 in Figure 2b), which corresponds to 39 samples and a time difference of $0.884 \mathrm{~ms}$.

We can understand better this fact if we take into account that the experiment is performed using a frequency of $2000 \mathrm{~Hz}$, that is, a period of $0.5 \mathrm{~ms}$. In this period of time, the sound travels about $17 \mathrm{~cm}$, so that, for a distance of $20 \mathrm{~cm}$ (example of Figure 2a) the measured time is approximately equal to one oscillation period. For a distance of $30 \mathrm{~cm}$ (example of Figure 2b) the measured time is greater than one oscillation period, although smaller than two oscillations periods.

The experiment consists of measuring the time difference between the signals for different distances between the speaker and the microphone. The distance is measured with a metric tape which has a precision of $0.001 \mathrm{~m}$. The measurements for the distances and time differences are plotted and fitted to the equation for the velocity in a uniform linear motion, $x=x_{0}+v t$, where the slope of the curve is the speed of sound in the air. It should be mentioned that the real distance between the emission point inside the microphone and the transmitter point inside the speaker is not exactly known. However, in our proposed procedure, this distance is not relevant, because it is related with the $x_{0}$ parameter in the previous equation, but not with the sound speed. When the data is fitted to this linear equation, the distance $x_{0}$ is the y-intercept. We only need the slope of the straight line to obtain the sound speed.

\section{Results}

By following the procedure aforementioned, the time difference between the speaker and microphone signals for 23 different distances between 0 and $110 \mathrm{~cm}$ (with an increment of $5 \mathrm{~cm}$ ) have been measured. Each measurement is repeated 3 times in order to evaluate the standard deviation (SD). For all cases, the SD is very small with respect to the direct error. The error in measuring the distance is the precision of the metric tape, that is, $0.001 \mathrm{~m}$, and in the measurement of time, $0.023 \mathrm{~ms}$, which corresponds to the sampling period $(1 / 44100 \mathrm{~Hz})$. The results of the experiments are presented in Figure 3, as well as the results of a least-squares linear fit. The resulting value of the slope corresponds to the speed of sound $(343 \pm 3) \mathrm{m} / \mathrm{s}$. The good quality of the fit is characterized by the linear correlation coefficient, $R=0.998$. The experiments were performed at room

temperature, $25.5^{\circ} \mathrm{C}$. The resulting value can be compared to the experimental value of reference [5] where authors performed a study of the speed of sound versus the air temperature yielding $347 \mathrm{~m} / \mathrm{s}$. The percentage discrepancy of our result with respect to the value of reference [5] is less than $0.02 \%$. 


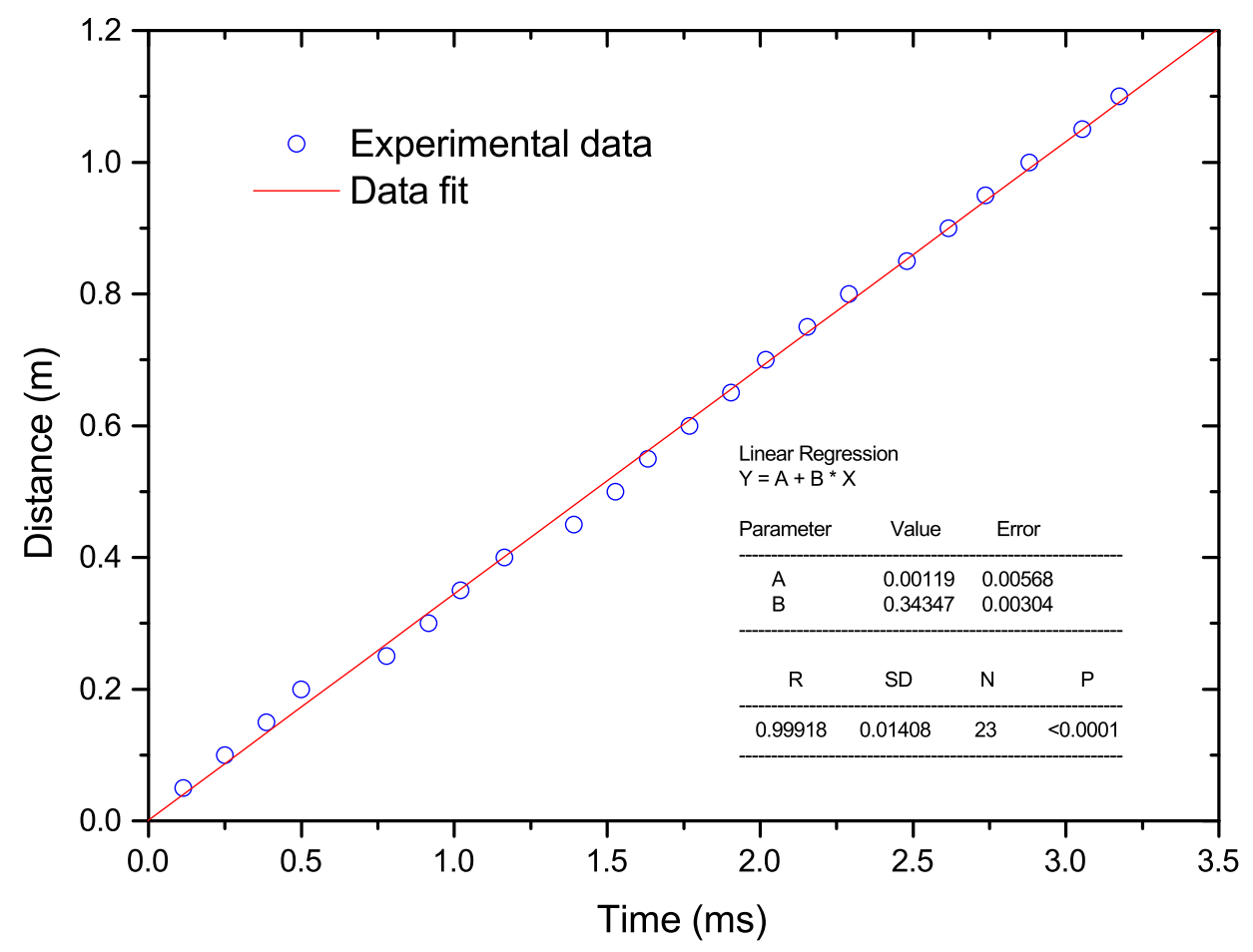

Figure 3. Distance between the speaker and the microphone versus the time-of-flight of the wave. The results of the fit are also included in the figure.

\section{Acknowledgments}

The authors would like to thank the Institute of Education Sciences, Universitat Politècnica de València (Spain), for the support of the Teaching Innovation Groups, MoMa and e-MACAFI.

\section{References}

[1] LoPresto M C 2011 Experimenting with end-correction and the speed of sound Phys. Educ. 46 437-439

[2] Ng Y and Mak S 2001 Measurement of the speed of sound in water Phys. Educ. 36 65-70

[3] Mak S, Ng Y and Wu K 2000 Measurement of the speed of sound in a metal rod Phys. Educ. 35 439-445

[4] Albergotti J C 1981 Speed of sound by a time-of-flight method Am. J. Phys. 49 595-596

[5] Ouseph P J, and Link J J 1984 Variation of speed of sound in air with temperature Am. J. Phys. $\mathbf{5 2} 661-661$

[6] http://audacity.sourceforge.net/ 